\title{
Increased Prevalence and Severity of Coronary Artery Calcification in Patients with Chronic Kidney Disease Stage III and IV
}

\author{
Maria Koukoulaki ${ }^{\mathrm{a}}$ Evangelos Papachristou ${ }^{\mathrm{a}}$ \\ Christina Kalogeropoulou ${ }^{\mathrm{b}}$ Maria Papathanasiouc $^{\mathrm{c}}$ \\ Petros Zampakis ${ }^{b}$ Maria Vardoulaki ${ }^{\mathrm{a}}$ Dimitrios Alexopoulos ${ }^{c}$ \\ Dimitrios S. Goumenos ${ }^{a}$
}

Departments of ${ }^{\mathrm{a}}$ Internal Medicine - Nephrology, ${ }^{\mathrm{b}}$ Radiology and ${ }^{\mathrm{c} C a r d i o l o g y}$, University Hospital of Patras, Patras, Greece

\section{Key Words}

Agatston score $\cdot$ Chronic kidney disease $\cdot$ Coronary artery calcification $\cdot$ Framingham risk

score $\cdot$ Multi-detector computed tomography $\cdot$ Parathormone

\begin{abstract}
Background: Cardiovascular disease (CVD) is the main cause of mortality in patients with chronic kidney disease (CKD). The pathophysiology of coronary artery disease in CKD is multifactorial including, in addition to traditional risk factors (hypertension, hyperlipidemia, diabetes mellitus), parameters related to uremia. Methods: The study consisted of measuring coronary artery calcification (CAC) score in patients with CKD stage III and IV without history of CVD and in a group of controls with normal renal function matched for age, gender and risk factors using multi-detector computed tomography. Results: The study included 49 patients and 49 controls. CAC was present in $79.6 \%$ in the CKD group versus $59.2 \%$ in the control group $(p=0.028)$. The median CAC score value in CKD patients was 139 (interquartile range (IQR): 23-321) versus 61 (IQR: 6-205) in controls $(p=0.007)$. CAC was associated with traditional risk factors such as older age, hypertension and baseline cardiovascular risk score, while CKD patients with severe calcification had marginally lower estimated glomerular filtration rate and increased levels of parathormone. Conclusions: CAC is more frequent and severe in patients with CKD stage III and IV compared to matched controls with normal renal function, even though kidney disease-related parameters are not directly correlated with intensity of calcification.
\end{abstract}

Copyright $\odot 2012$ S. Karger AG, Basel 


\section{Introduction}

Cardiovascular disease (CVD) is the main cause of mortality in patients with chronic kidney disease (CKD) (US Renal Data System: USRDS 2009 Annual Data Report). The pathophysiology of coronary artery disease in CKD is multifactorial including, next to traditional risk factors such as older age, diabetes mellitus, systolic hypertension, left ventricular hypertrophy and hyperlipidemia, parameters related to CKD such as chronic inflammation, oxidative stress, abnormal bone and mineral metabolism, hyperhomocysteinemia, malnutrition and anemia $[1,2]$. Although it is questionable how the earlier stages of kidney disease are associated with and affected by these non-traditional CVD risk factors, epidemiological studies have concluded that even patients with early stages of CKD have an increased risk for cardiovascular events and related mortality [3-5]. The natural history of CKD was analyzed by Keith et al. [6] in a population of 27,998 patients with impaired renal function (CKD stages II-V). They concluded that death was far more common than dialysis at all stages. Therefore assessment of CVD in patients with declining renal function is essential.

Coronary artery calcification (CAC) has been identified as a strong predictor of CVD progression and patient survival in patients without kidney disease $[7,8]$ since arterial calcium deposition is involved in the creation and evolution of atherosclerotic plaque [9]. Computed tomography has been successfully used to quantify CAC [10]. The calculated CAC score displays very high sensitivity and negative predictive value for both obstructive and non-obstructive coronary artery disease [7]. CAC in various stages of CKD has been investigated either as part of large observational, epidemiological studies or in cohort studies reporting varying prevalence [11-16]. We performed this study to evaluate CAC score in patients with CKD stage III and IV and matched controls with normal renal function using multi-detector computed tomography (MDCT). Primary endpoint was the comparison of CAC score between CKD patients and controls and secondly the evaluation of associations of CAC score with traditional CVD risks factors and non-traditional risk factors related to CKD.

\section{Methods}

\section{Patient and Control Selection}

We conducted a prospective single-center study measuring CAC score in patients with CKD stage III and IV and in a group of controls who did not suffer from CKD using MDCT. The study was approved by the Local Bioethics Committee and all participants provided written informed consent.

Patients were selected from the Outpatient Renal Clinic. Subjects $>18$ years of age with CKD stage III or IV were included in the study. Patients with documented CVD (history of acute myocardial infarction, angina pectoris, and/or ischemia on provocative test), diabetes mellitus and patients who had been previously treated with immunosuppressive agents (such as corticosteroids or cyclosporine) were excluded from the study. Controls were selected from volunteers without history of heart disease or diabetes who were screened at the Outpatient Cardiology Clinic and were submitted to MDCT. History, clinical examination and laboratory tests of blood and urine were performed to evaluate renal function and exclude subjects with CKD. Controls were matched to patients based both on their demographic characteristics (age and gender) and risk factors (smoking, hypertension and hyperlipidemia). 


\section{Clinical and Laboratory Data Selection}

Data on demographic characteristics were collected regarding age, gender, height, weight, body mass index, diagnosis and duration of renal disease. Baseline biochemical parameters were measured using routine laboratory methods. Data obtained included serum creatinine, urea, alkaline phosphatase, total cholesterol, low-density lipoprotein cholesterol, high-density lipoprotein cholesterol, serum calcium, phosphorus and intact parathormone (PTH). The four-variable Modification of Diet in Renal Disease (MDRD) formula was used to estimate glomerular filtration rate (eGFR) [17]. National Kidney Foundation (NKF) criteria were applied to diagnose CKD stage III (eGFR: $30-59 \mathrm{ml} / \mathrm{min} / 1.73 \mathrm{~m}^{2}$ ) and IV (eGFR: $15-29 \mathrm{ml} / \mathrm{min} / 1.73 \mathrm{~m}^{2}$ ) [18]. In CKD stage III PTH should remain within normal limits (35$70 \mathrm{pg} / \mathrm{ml}$ ) while in CKD stage IV PTH could vary from 70 to $110 \mathrm{pg} / \mathrm{ml}$ [19]. Risk factors such as smoking, hypertension and hyperlipidemia were recorded. Hypertension was defined as systolic blood pressure $>140 \mathrm{~mm} \mathrm{Hg}$ and/or diastolic blood pressure $>90 \mathrm{~mm} \mathrm{Hg}$ or treatment with antihypertensive medication. Hyperlipidemia was considered as low-density lipoprotein cholesterol level $>100 \mathrm{mg} / \mathrm{dl}$ requiring lipid-lowering treatment [20]. Cardiovascular risk score or Framingham risk score was determined based on traditional risk factors for CVD [21].

\section{MDCT Protocol for CAC Score Measurements}

All patients were examined in a 16-MDCT scanner (LightSpeed 16×; General Electric, Milwaukee, Wisc., USA) with prospective electrocardiographic triggering during a single breath hold (typically $8 \mathrm{~s}$ ). Data were obtained from the entire heart using sequential acquisition. Scans were prospectively initiated at $50 \%$ of the RR interval, which has been widely used for MDCT-based measurements of CAC and has been shown to provide the best average image quality for MDCT-based data acquisition [22]. Forty-eight contiguous 2.5-mmthick slices $(120 \mathrm{kV}, 250 \mathrm{~mA}$ and gantry rotation time $0.5 \mathrm{~s}$ and exposure time per rotation $0.3 \mathrm{~s}$ ) were obtained. CT scans were evaluated by an experienced radiologist for the presence and amount of CAC using a dedicated workstation (Advantage Workstation 4.2). Quantification of CAC was provided by a computerized program (SmartScore 3.5, GE Medical Systems, Milwaukee, Wisc., USA) that calculated the CAC score as Agatston score. A calcified lesion was defined as an area equal or above 3 connected pixels with an attenuation above 130 Hounsfield Units applying three-dimensional connectivity criteria (6 points). CAC score was calculated by multiplying the area of each lesion with a weighted attenuation score dependent on the maximal attenuation within the lesion [10].

Based on CAC score measurements, 5 distinct categories were classified as follows: no evidence of CAC (CAC score $=0)$, minimal CAC (CAC score $=1-10)$, mild CAC (CAC score $=11-100)$, moderate CAC $($ CAC score $=101-400)$, and severe CAC $($ CAC score $>400)$.

\section{Statistical Analysis}

Normally distributed variables were expressed as mean \pm standard deviation (SD), while skewed continuous variables were described as median value and interquartile range (IQR). Statistical analysis of categorical characteristics was performed using $\chi^{2}$ test, Fischer's exact test and for ordinal parameters using Spearman's R. Student's t test and Mann-Whitney $\mathrm{U}$ test were applied accordingly to identify differences in continuous parametric and nonparametric variables. Prior to analysis parameters without normal distribution were logarithmically transformed to ensure normality. Bivariate analysis with Pearson correlation was applied to identify associations between continuous variables. The value 1 was added to CAC score values prior to log transformation. 
Patients with Chronic Kidney Disease Stage III and IV

Table 1. Demographic characteristics and risk factors of study participants

\begin{tabular}{llll}
\hline & Patients & Controls & $\mathrm{p}$ \\
\hline Total number of participants & 49 & 49 & \\
Mean age \pm SD, years & $61.3 \pm 13.2$ & $58.4 \pm 5.5$ & 0.4 \\
Gender, male/female & $23 / 26$ & $23 / 26$ & 1 \\
Smokers & $15(30.6 \%)$ & $19(38.8 \%)$ & 0.6 \\
Hypertension & $38(77.6 \%)$ & $34(65.3 \%)$ & 0.7 \\
Hyperlipidemia & $24(49 \%)$ & $20(40.8 \%)$ & 0.7 \\
Stage of CKD, III/IV & $28 / 21$ & & $<0.001$ \\
Mean eGFR \pm SD, $\mathrm{ml} / \mathrm{min} / 1.73 \mathrm{~m}^{2}$ & $32.3 \pm 12.5$ & $84.2 \pm 9.3$ & $<$ \\
\hline
\end{tabular}

Table 2. Etiology of CKD

\begin{tabular}{ll}
\hline Etiology & Number $(\%)$ \\
\hline Nephrolithiasis & $11(22.5)$ \\
Glomerular disease & $10(20.4)$ \\
Polycystic kidney disease & $6(12.2)$ \\
Hypertension & $6(12.2)$ \\
Unilateral kidney & $5(10.2)$ \\
Tubulointerstitial nephritis & $3(6.1)$ \\
Obstructive nephropathy & $1(2.1)$ \\
Unknown & $7(14.3)$ \\
\hline
\end{tabular}

\section{Results}

Demographic Characteristics, Risk Factors and Laboratory Parameters

The study included 49 patients ( 23 men/26 women, aged $61.3 \pm 13.2$ years) with CKD stage III or IV and 49 controls with normal renal function matched for age, gender and risk factors (23 men/26 women, aged $58.4 \pm 5.5$ years). Demographic characteristics and risk factors are presented for both groups in table 1. The etiology of CKD is presented in table 2. The Framingham risk score in the group of CKD patients was $11.2 \pm 7.8 \%$. Low $(<10 \%)$, moderate (10-20\%) and high (>20\%) Framingham risk scores were found in 44.9\% (22/49), $38.8 \%(19 / 49)$ and $16.3 \%$ (8/49) patients, respectively. Patients' body mass index was $22.34 \pm$ $2.98 \mathrm{~kg} / \mathrm{m}^{2}$ and none had a body mass index $>30 \mathrm{~kg} / \mathrm{m}^{2}$. Mean duration of CKD was $7.1 \pm$ 5.3 years. Mean eGFR in CKD patients and controls was $32.3 \pm 12.5 \mathrm{ml} / \mathrm{min} / 1.73 \mathrm{~m}^{2}$ and $84.2 \pm 9.3 \mathrm{ml} / \mathrm{min} / 1.73 \mathrm{~m}^{2}$, correspondingly $(\mathrm{p}<0.001)$.

Based on NKF criteria, 28 patients (57.2\%) had CKD stage III with a mean eGFR of 41 $\pm 8.5 \mathrm{ml} / \mathrm{min} / 1.73 \mathrm{~m}^{2}$ and 21 patients $(42.8 \%)$ were classified as CKD stage IV with a mean eGFR of $20.7 \pm 5.6 \mathrm{ml} / \mathrm{min} / 1.73 \mathrm{~m}^{2}$. Biochemical examination showed mean serum calcium of $9.5 \pm 0.62 \mathrm{mg} / \mathrm{dl}$, mean serum phosphorus of $3.8 \pm 0.78 \mathrm{mg} / \mathrm{dl}$, and the calciumphosphorus product $(\mathrm{Ca} \times \mathrm{P})$ was $36.57 \pm 7.3 \mathrm{mg}^{2} / \mathrm{dl}^{2}$. A small proportion of patients $(7 / 49,14.3 \%)$ displayed increased values of $\mathrm{Ca} \times \mathrm{P}>45 \mathrm{mg}^{2} / \mathrm{dl}^{2}$. Mean intact PTH was 123 $\pm 105 \mathrm{pg} / \mathrm{ml}$ and exceeded the accepted level in 26 patients, of whom 12 had CKD stage III and 14 CKD stage IV. Results from all examined laboratory parameters are displayed in table 3. 
Fig. 1. CAC score in CKD. Box plots of CAC score in patients with CKD (stage III and IV) and matched controls. The number next to the line in the box plots indicates the median value of the data. The $\mathrm{p}$ value indicates the statistical significance of comparison of CAC scores between CKD patients and controls. ${ }^{\circ}$ Outlier (a value between 1.5 and 3 times the interquartile range from a quartile). * Extreme outlier (a value more than 3 times the interquartile range from a quartile.

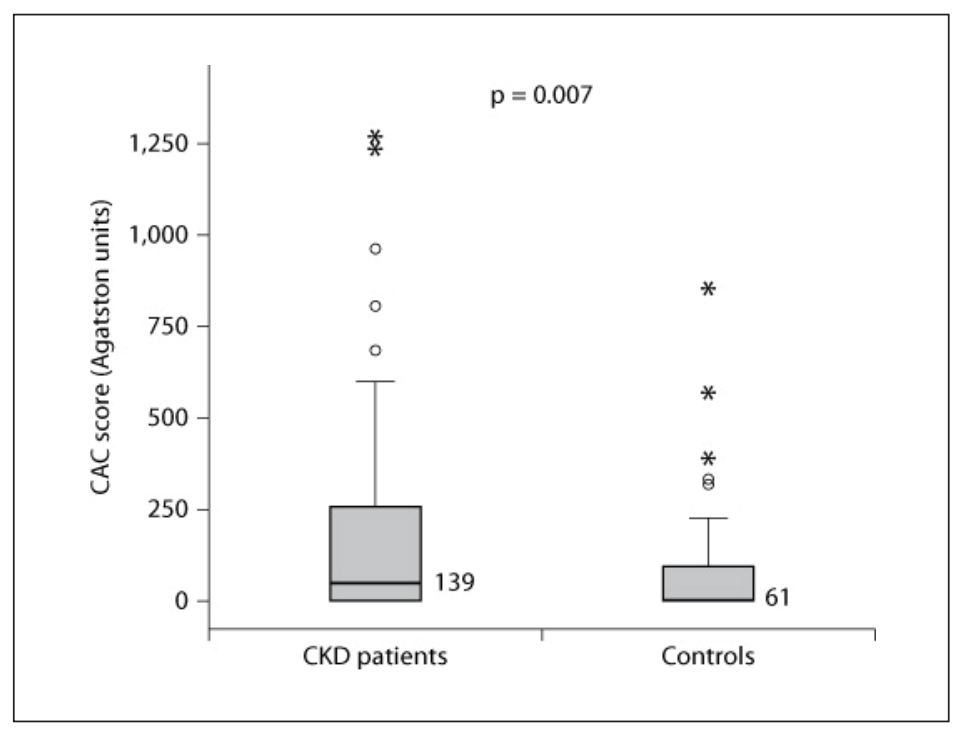

Table 3. Laboratory parameters regarding renal function and bone mineral metabolism in patients with CKD stage III and IV

\begin{tabular}{|c|c|c|c|}
\hline Variable & All CKD patients & Stage III & Stage IV \\
\hline Serum urea, mg/dl & $89.22 \pm 39.59$ & $64.89 \pm 19.16$ & $121.67 \pm 36.49$ \\
\hline Serum creatinine, $\mathrm{mg} / \mathrm{dl}$ & $2.29 \pm 0.93$ & $1.7 \pm 0.32$ & $3.08 \pm 0.9$ \\
\hline $\mathrm{eGFR}, \mathrm{ml} / \mathrm{min} / 1.73 \mathrm{~m}^{2}$ & $32.32 \pm 12.52$ & $41.03 \pm 8.49$ & $20.7 \pm 5.6$ \\
\hline Serum calcium, mg/dl & $9.56 \pm 0.62$ & $9.7 \pm 0.59$ & $9.37 \pm 0.61$ \\
\hline Serum phosphorus, mg/dl & $3.84 \pm 0.78$ & $3.57 \pm 0.77$ & $4.19 \pm 0.67$ \\
\hline $\mathrm{Ca} \times \mathrm{P}, \mathrm{mg}^{2} / \mathrm{dl}^{2}$ & $36.57 \pm 7.31$ & $34.51 \pm 6.81$ & $39.3 \pm 7.2$ \\
\hline Alkaline phosphatase, U/l & $114.1 \pm 65.3$ & $124.25 \pm 76.12$ & $100.57 \pm 54.56$ \\
\hline Intact PTH, pg/ml & $123.3 \pm 105.5$ & $85.96 \pm 99.6$ & $173 \pm 93.55$ \\
\hline Cholesterol, mg/dl & $194.74 \pm 32.63$ & $199.81 \pm 31.23$ & $187.9 \pm 34.03$ \\
\hline HDL cholesterol, mg/dl & $52.11 \pm 12.99$ & $51.85 \pm 14.11$ & $52.451 \pm 11.66$ \\
\hline LDL cholesterol, mg/dl & $112.62 \pm 29.95$ & $116.85 \pm 31.45$ & $106.9 \pm 27.53$ \\
\hline
\end{tabular}

Values are expressed as mean $\pm \mathrm{SD}$. HDL = High-density lipoprotein; LDL = low-density lipoprotein.

\section{CAC Score in Patients and Controls}

CAC was present (CAC score >0) in 79.6\% (39/49) of patients from the CKD group and $59.2 \%(29 / 49)$ of patients from the control group $(\mathrm{p}=0.028)$. The median CAC score value in CKD patients was 139 (IQR: 23-321) versus 61 (IQR: 6-205) in controls, which shows that CAC scores differed significantly $(\mathrm{p}=0.007)$ (fig. 1$)$.

More specifically, in the group of patients with CKD, 12.2\% (6/49) had minimal calcification (median CAC score: 1.5, IQR: 1-4.75), 22.4\% (11/49) had mild calcification (median CAC score: 29, IQR: $18-46), 30.6 \%$ (15/49) moderate calcification (median CAC score: 219, IQR: 156-281) and $14.3 \%$ (7/49) presented severe calcification (median CAC score: 807, IQR: $600-$ 1,234). In the control group, $16.3 \%$ had minimal calcification with a median CAC score of 5 (IQR: 1.5-5.75), 18.4\% (9/49) showed mild calcification (median CAC score: 34, IQR: 17-61), 20.4\% (10/49) had moderate calcification (median CAC score: 205, IQR: 147-322) and just $4.1 \%(2 / 49)$ had severe calcification. The data are presented in figure 2 and tabulated in table 4. 
Patients with Chronic Kidney Disease Stage III and IV

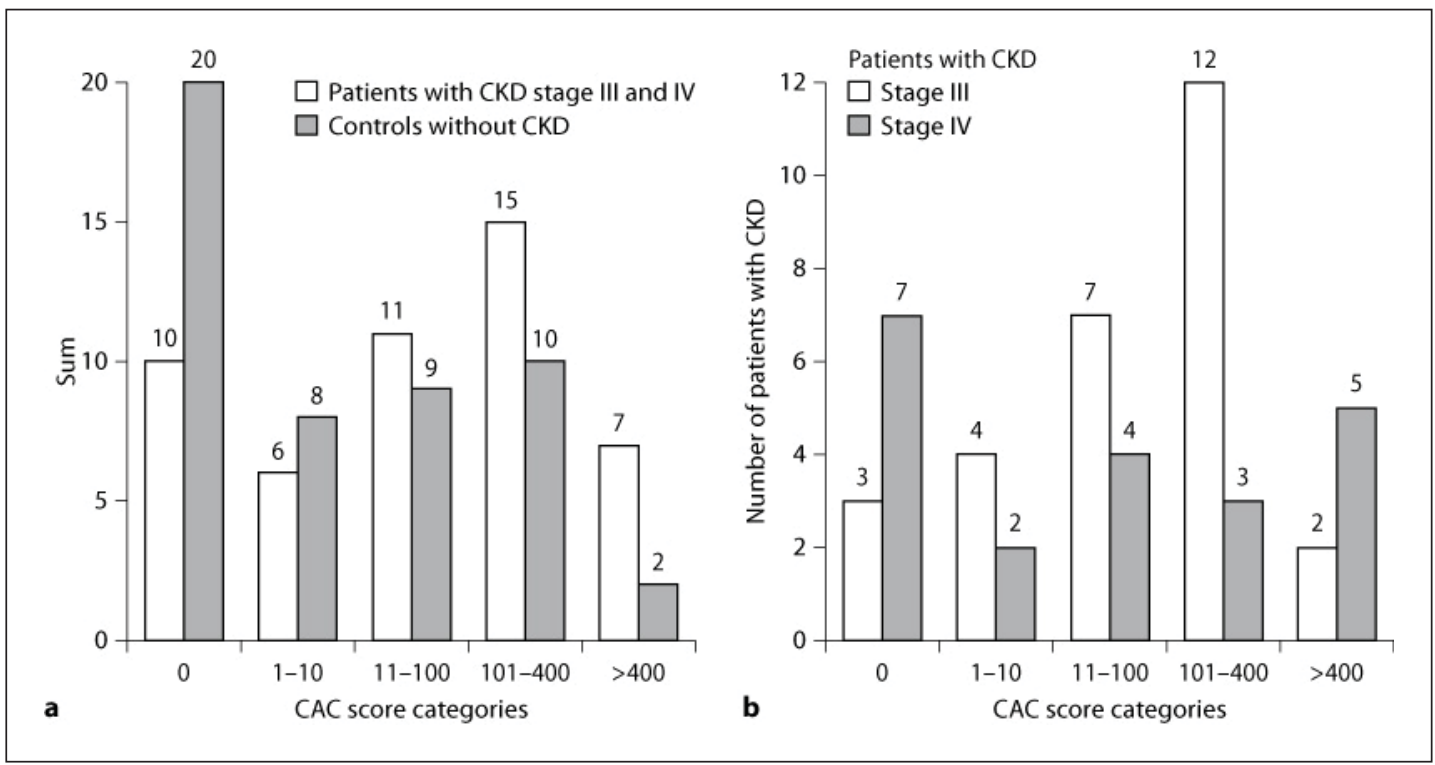

Fig. 2. CAC score categorization in patients with CKD stage III and IV and controls without CKD. a Patients with CKD and controls without CKD. b Patients with CKD stage III and IV categorized based on CAC score measurements. Numbers above bars represent the sum of either patients or controls, with CAC score measurements corresponding to the relevant category.

Table 4. CAC in patients with CKD stage III and IV and controls without CKD

\begin{tabular}{lccc}
\hline & Patients with CKD & Controls & $\mathrm{p}$ \\
\hline No evidence of calcification (CAC score $=0)$ & $10(20.4 \%)$ & $20(40.8 \%)$ & 0.02 \\
Presence of calcification (CAC score $>0)$ & $39(79.6 \%)$ & $29(59.2 \%)$ & 0.02 \\
$\quad$ Median (IQR) & $139(23-321)$ & $61(6-205)$ & 0.007 \\
Minimal calcification (CAC score = 1-10) & $6(12.2 \%)$ & $8(16.3 \%)$ & 0.5 \\
$\quad$ Median (IQR) & $1.5(1-4.75)$ & $5(1.5-5.75)$ & \\
Mild calcification (CAC score $=11-100)$ & $11(22.4 \%)$ & $9(18.4 \%)$ & 0.6 \\
$\quad$ Median (IQR) & $29(18-46)$ & $34(17-61)$ & \\
Moderate calcification (CAC score = 101-400) & $15(30.6 \%)$ & $10(20.4 \%)$ & 0.1 \\
$\quad$ Median (IQR) & $219(156-281)$ & $205(147-322)$ & \\
Severe calcification (CAC score $>400)$ & $7(14.3 \%)$ & $2(4.1 \%)$ & 0.05 \\
$\quad$ Median (IQR) & $807(600-1234)$ & $710(568-853)$ & \\
\hline
\end{tabular}

\section{CAC Score and Traditional Risk Factors in CKD Patients}

CAC score was evaluated in relation to traditional risk factors in patients with CKD (table 5). CAC score ( $\log )$ was positively correlated with age $(\mathrm{r}=0.48, \mathrm{p}<0.001)$ (fig. 3a). The mean value of age (61 years) was used to compare CAC scores between two age groups. Older patients $>61$ years old had significantly higher CAC scores $(\mathrm{p}=0.003)$ (fig. 3b). Although CAC score measurements showed a borderline difference between hypertensive and nonhypertensive patients $(\mathrm{p}=0.06)$, patients with hypertension had a significantly higher incidence of CAC ( $\mathrm{p}=0.02)$. In addition, CAC did not correlate with risk factors of gender $(\mathrm{p}=$ $0.1)$, smoking $(p=0.1)$ and hyperlipidemia $(p=0.9)$. CAC scores did not differ between males and females $(\mathrm{p}=0.4)$, smokers and non-smokers $(\mathrm{p}=0.7)$ and patients with or without hyperlipidemia $(\mathrm{p}=0.8)$. 


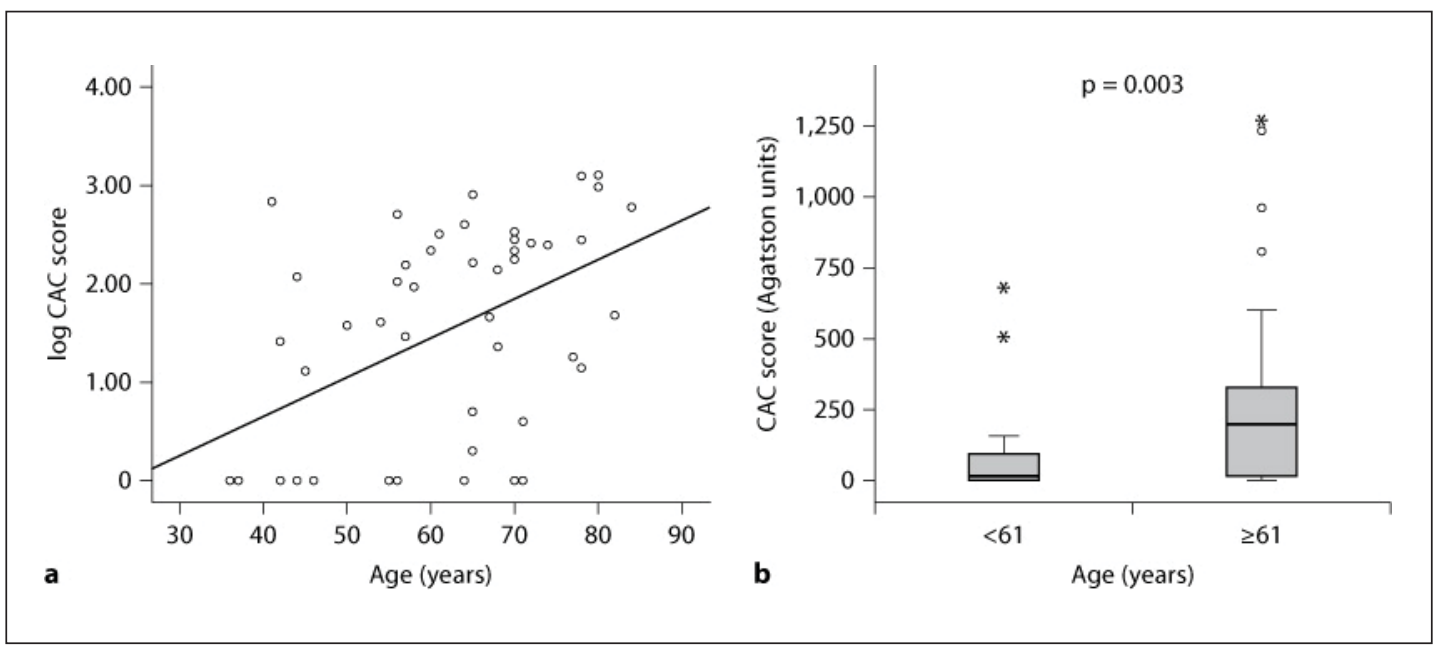

Fig. 3. Correlation of CAC score with age. a Scatter plot of log CAC score and age in patients with CKD (stage III and IV). b Box plots of CAC scores of patients with CKD (stage III and IV) according to their age group. The $\mathrm{p}$ value indicates the statistical significance of comparison of CAC scores between the two age groups. ${ }^{\circ}$ Outlier (a value between 1.5 and 3 times the interquartile range from a quartile). ${ }^{*}$ Extreme outlier (a value more than 3 times the interquartile range from a quartile.

Table 5. Associations of CAC with traditional risk factors

\begin{tabular}{lll}
\hline & Presence of CAC & CAC score \\
\hline Age & $\mathrm{p}=0.001$ & $\mathrm{r}=0.48, \mathrm{p}<0.001$ \\
Gender & $\mathrm{p}=0.1$ & $\mathrm{p}=0.4$ \\
Smoking & $\mathrm{p}=0.1$ & $\mathrm{p}=0.7$ \\
Hypertension & $\mathrm{p}=0.02$ & $\mathrm{p}=0.06$ \\
Hyperlipidemia & $\mathrm{p}=0.9$ & $\mathrm{p}=0.7$ \\
Framingham risk score & $\mathrm{p}<0.001$ & $\mathrm{r}=0.518, \mathrm{p}<0.001$ \\
\hline
\end{tabular}

However, the cardiovascular risk score (Framingham risk score) was strongly correlated both with the presence $(\mathrm{p}<0.001)$ (fig. $4 \mathrm{a})$ and the severity of calcification $(\mathrm{r}=0.518, \mathrm{p}<$ $0.001)$. Median CAC scores (IQR) in patients with low (<10\%), moderate (10-20\%) and high Framingham risk scores (>20\%) were 1 (1-141.1), 118 (18-248) and 281 (93-1,234), respectively, showing a significant increase as the cardiovascular risk score rose $(\mathrm{p}=0.008)$ (fig. $4 \mathrm{~b})$. It should be further noted that all patients with moderate and high Framingham risk scores had evidence of CAC, while CAC was present in 54.5\% (12/22) of patients with low Framingham risk scores.

\section{CAC Score and Risk Factors Related to CKD and Mineral and Bone Disease}

The potential associations of CAC with CKD-related parameters were analyzed. Prevalence of calcification and CAC score did not differ between patients with CKD stage III and IV. In detail, $89.3 \%$ of patients $(25 / 28)$ with CKD stage III and $66.7 \%$ of patients (14/21) with CKD stage IV had positive results for CAC $(\mathrm{p}=0.06)$ and median scores were $99(7-240)$ and $26(1-423)$ for each CKD stage $(\mathrm{p}=0.6)$. In addition, a decline of eGFR did not show linear correlation with an increase of CAC score $(\mathrm{r}=0.058, \mathrm{p}=0.7)$. Duration of CKD was not associated with CAC $(\mathrm{p}=0.1)$. 


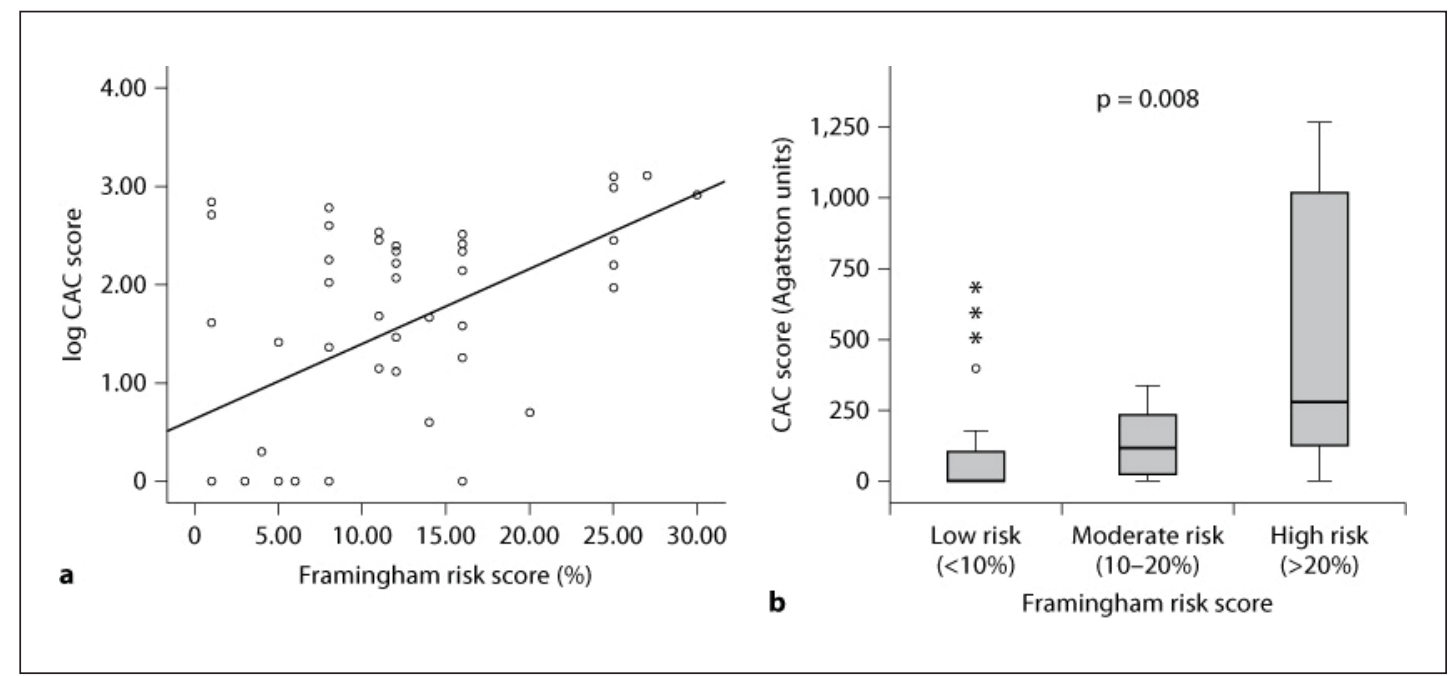

Fig. 4. Correlation of CAC score with Framingham risk score. a Scatter plot of log CAC score and Framingham risk score in patients with CKD (stage III and IV). $\mathbf{b}$ Box plots of CAC scores of patients with CKD (stage III and IV) according to the rank of Framingham risk score. The p value indicates the statistical significance of comparison of CAC score between patients with low, moderate and high Framingham risk scores. ${ }^{\circ}$ Outlier (a value between 1.5 and 3 times the interquartile range from a quartile). * Extreme outlier (a value more than 3 times the interquartile range from a quartile.

Table 6. Bivariate analysis of correlations between CAC score and parameters related to $\mathrm{CKD}$ and mineral bone disease

\begin{tabular}{lll}
\hline Variables & Pearson correlation, $\mathrm{r}$ & $\mathrm{p}$ \\
\hline eGFR & 0.058 & 0.7 \\
Serum calcium & 0.208 & 0.1 \\
Serum phosphorus & 0.033 & 0.8 \\
Ca $\times$ P & 0.102 & 0.4 \\
PTH & 0.06 & 0.6 \\
Alkaline phosphatase & 0.132 & 0.3 \\
Cholesterol & -0.131 & 0.3 \\
HDL cholesterol & -0.085 & 0.5 \\
LDL cholesterol & -0.159 & 0.2 \\
\hline
\end{tabular}

HDL $=$ High-density lipoprotein LDL = low-density lipoprotein.

Bivariate analysis of CAC score measurements (log CAC) and the examined laboratory variables including eGFR and parameters related to bone and mineral metabolism (serum calcium, serum phosphorus, calcium - phosphorus product and PTH) showed no significant correlation, as is displayed in table 6.

However, patients with severe calcification (CAC score >400) had lower eGFR, although the difference did not reach statistical significance $(\mathrm{p}=0.09)$, and considerably higher blood PTH levels $(\mathrm{p}=0.007)$ (fig. 5). 
Fig. 5. Correlation of CAC score with PTH. Error bars of intact PTH in patients with CKD and CAC score $<400$ and in patients with CKD and severe CAC (CAC score $>400)$. The $\mathrm{p}$ value indicates the statistical significance of comparison of PTH between CKD patients with CAC score $<400$ or $>400$.
Koukoulaki et al.: Increased Prevalence and Severity of Coronary Artery Calcification in

Patients with Chronic Kidney Disease Stage III and IV

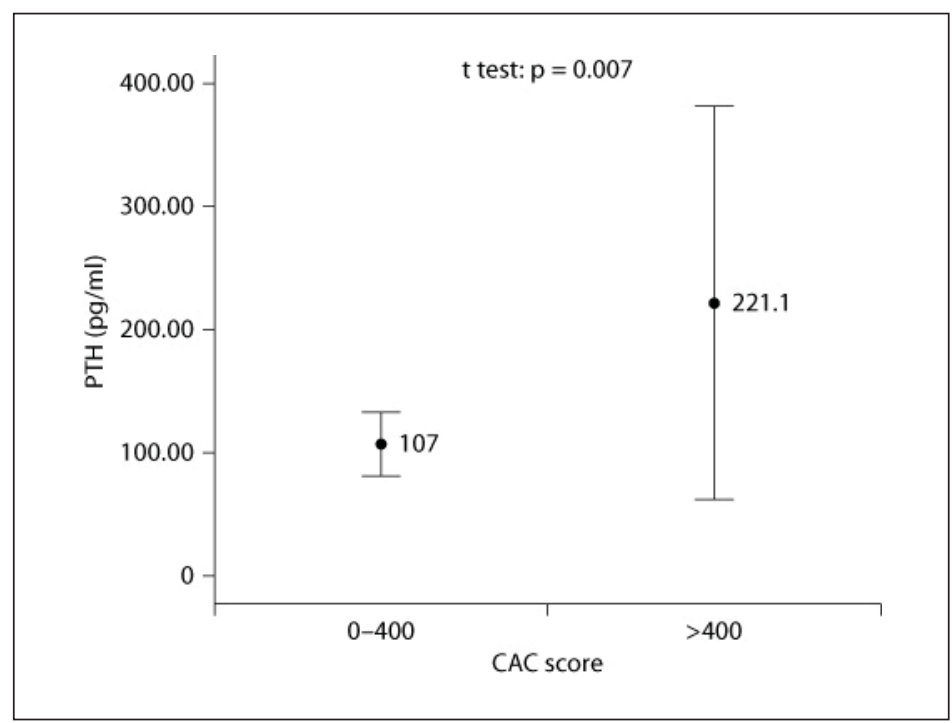

\section{Discussion}

Cardiovascular calcification is considered highly predictive of cardiovascular mortality and morbidity. Imaging of CAC by computed tomography and calculation of CAC score has been proven sensitive in diagnosing coronary artery disease $[7,8,10]$. This study evaluated CAC using MDCT in patients with moderate and severe kidney disease (CKD stage III and IV) in comparison to matched controls with normal renal function.

It is well established that patients on hemodialysis and peritoneal dialysis are at greater risk for cardiovascular calcification [23-26]. The prevalence of CAC in earlier stages of kidney disease varies from $13.9 \%$ in stage I and II up to $83 \%$ in stages III-V. In this study, the prevalence of CAC in patients with moderate and severe CKD without documented CVD was $79.6 \%$. CAC was identified in $89.3 \%$ of patients with CKD stage III and $66.7 \%$ of patients with CKD stage IV. Our results agree with previously published data. Piers et al. [16] examined the effect of CKD stage III, IV and V on CAC. CAC was observed in $67 \%$ of participants and corresponded to $77 \%$ of patients in CKD stage III, 54\% in CKD stage IV and 64\% in CKD stage V. However, no correlation of CAC with stage of CKD was evident. Garland et al. [15] reported an evidence of $\mathrm{CAC}$ of $83.2 \%$ in a population comparable to ours with $\mathrm{CKD}$ stage III-V (excluding dialysis) without known CVD but including diabetic patients too. Earlier studies by Russo et al. [14] and Tomiyama et al. [27] have reported a lower incidence of CAC (40 and 64\%, respectively) in patients with impaired renal function. This is probably due to the younger age of participants and the inclusion of patients with CKD stage II. Supporting evidence is found in a study of 101 kidney donors classified as CKD stage I and II, of whom only $13.9 \%$ presented CAC [28]. It is obvious that even if cardiovascular calcification is not very frequently observed in patients with mild renal function impairment, the prevalence of $\mathrm{CAC}$ in moderate and severe kidney disease is increasing, reaching that of end-stage renal disease. Conflicting evidence supports the relation between CAC and declining creatinine clearance. Fox et al. [29] reported that in a cohort of subjects free of symptomatic CVD, GFR is inversely associated with CAC. However, other investigators have not reproduced these results $[14,15]$. In the Multi-Ethnic Study of Atherosclerosis, initially kidney dysfunction was associated with CAC but associations were not preserved after adjustment for demographic parameters (age, gender, race) or hypertension [12]. Even though we did not find a correla- 
tion between renal function and CAC, with the limitation of the restricted number of participants, we observed that patients with severe calcifications had lower eGFR.

In our study patients with history of diabetes mellitus and treatment with immunosuppression were excluded in an attempt to isolate kidney disease as a risk factor for CAC. It has been shown that diabetic patients even without kidney disease have higher CAC scores [11]. Furthermore, severe calcifications are identified in patients with diabetic nephropathy and advanced CKD (stage IV and V) [30]. In a representative sample of diabetic participants in the Dallas Heart Study, CKD stage III-V was associated with an eightfold increased odds of severe calcifications (CAC score $>400$ ) versus minimal calcifications (CAC score $<10$ ) compared to a population without CKD [11]. In addition, both corticosteroids and cyclosporine aggravate hypertension and dyslipidemia, having an adverse effect on long-term cardiovascular risk [31].

Epidemiological studies have brought to attention that CKD patients even at the earlier stages have an increased incidence of cardiovascular events compared to the rest of the population [3, 4]. Studies on CAC have verified this observation. Previous studies have looked into differences in CAC between CKD and healthy populations without considering risk factors of the control population. Published data by Russo et al. [14] confirm the difference in CAC between CKD patients and healthy controls, while Seyahi et al. [28] did not find a difference between patients with CKD I and II and normal controls. No other studies have analyzed patients in comparison to matched controls. We matched individuals with normal renal function to patients with CKD III and IV in an attempt to isolate kidney disease as risk factor. For this reason, controls were paired to CKD patients based on demographic characteristics (age and gender) and traditional risk factors (smoking, hypertension and hyperlipidemia). Almost $60 \%$ of our control group presented CAC that could be attributed to the methodology to select subjects without history or symptoms of CVD, who had nonetheless risk factors for CVD progression, e.g. hyperlipidemia, smoking and most importantly hypertension, in proportions equivalent to that of the CKD group. Therefore, the CKD parameter could be considered as the discriminating factor between the examined populations, highlighting that in CKD patients prevalence of CAC and intensity of calcifications are greater.

Our analysis showed that CAC score was positively correlated with age and hypertension. Association of CAC with older age has been a constant finding in a lot of studies [15, 16]. Even though gender, smoking and hyperlipidemia were not independently linked with presence or severity of calcification probably due to sample size, the risk score of 10 -year coronary heart disease (Framingham risk score) that takes into account all the major traditional risk factors including decade of age, gender, smoking, systolic blood pressure, treatment for hypertension, level of total cholesterol and low-density lipoprotein, was strongly associated with CAC [21]. However, the predictive value of the Framingham risk score has been questioned when it is applied in CKD patients [32]. In our study the purpose of its use was to depict the cardiovascular risk in addition to separate risk factors. An increase of the Framingham risk score was accompanied by more extensive calcifications.

Disorders of bone and mineral metabolism in CKD have been related to an increased risk of coronary and peripheral artery calcifications especially in patients with end-stage kidney disease on hemodialysis [33]. However, patients with earlier stages of kidney disease who have not been exposed to dialysis-related risk factors have considerable calcifications. Our analysis did not reveal significant associations between CAC and kidney disease-related parameters such as duration of renal impairment or stage of CKD. More specifically, a higher prevalence of CAC in CKD stage III was noted, but it was not statistically significant ( $\mathrm{p}=$ 0.06). It should be noted though that 4 out of 7 patients with CKD stage IV without CAC were women of young age (range 28-40 years). It is rational to assume that CAC will evolve with progression of CKD, but it is not a univariate relationship. There is consensus that the etiol- 
ogy of coronary artery disease is multifactorial, depending on traditional risk factors but also on parameters related to CKD in this population.

Furthermore, the evaluation of CAC with parameters of mineral metabolism did not reveal any significant correlation. However, significantly higher blood PTH levels were observed in the subgroup of patients with severe CAC. Experimental data has implicated that PTH could contribute to the atherosclerotic process. The upregulation and expression of endothelial parameters involved in vascular calcification was shown both in vitro and in vivo following continuous stimulation or infusion of PTH $[34,35]$. In humans, evidence supports greater CVD risk in primary hyperparathyroidism, and further observational and epidemiological studies have linked increased PTH levels (above the normal limit) to left ventricular hypertrophy and cardiovascular morbidity and mortality [36-38]. However, it would be considerate to evaluate PTH regulation, an integral part of bone and mineral metabolism, in conjunction to serum calcium and phosphorus since serum phosphorus has also been identified as an independent factor of the presence and progression of vascular calcification. A separate analysis from the Multi-Ethnic Study of Atherosclerosis concluded that serum phosphate concentrations even within the normal laboratory range were associated with increased prevalence of vascular calcification in subjects with moderate CKD without documented CVD [13]. In addition, a prospective cohort analysis from the Spokane Heart Study reported that higher levels of serum phosphorus predicted evolution of CAC [39].

It should be acknowledged that our study results may be limited by the relatively restricted sample size and would certainly benefit from the extension of this research to a larger group of CKD patients and matched controls.

In conclusion, patients with CKD stage III and IV have a higher prevalence and severity of CAC compared to matched controls. Although CAC was not strongly associated with risk factors related to kidney disease, presence of renal function impairment and secondary hyperparathyroidism might contribute, along with traditional risk factors, to the development of CAC. Further research is required in order to identify the influence of early stages of CKD in the vascular calcification of coronary and peripheral arteries. It is essential that preventive strategies are investigated in an attempt to reduce all potential risk factors of CVD in patients with CKD.

\section{References}

1 Sarnak MJ, Levey AS: Cardiovascular disease and chronic renal disease: a new paradigm. Am J Kidney Dis 2000;35:S117-S131.

-2 Sarnak MJ, Levey AS, Schoolwerth AC, et al: Kidney disease as a risk factor for development of cardiovascular disease: a statement from the American Heart Association Councils on Kidney in Cardiovascular Disease, High Blood Pressure Research, Clinical Cardiology, and Epidemiology and Prevention. Circulation 2003;108:2154-2169.

-3 Weiner DE, Tighiouart H, Amin MG, et al: Chronic kidney disease as a risk factor for cardiovascular disease and all-cause mortality: a pooled analysis of community-based studies. J Am Soc Nephrol 2004; 15:1307-1315.

-4 Di Angelantonio E, Chowdhury R, Sarwar N, Aspelund T, Danesh J, Gudnason V: Chronic kidney disease and risk of major cardiovascular disease and non-vascular mortality: prospective population based cohort study. BMJ 2010;341:c4986.

-5 Vanholder R, Massy Z, Argiles A, Spasovski G, Verbeke F, Lameire N: Chronic kidney disease as cause of cardiovascular morbidity and mortality. Nephrol Dial Transplant 2005;20:1048-1056.

-6 Keith DS, Nichols GA, Gullion CM, Brown JB, Smith DH: Longitudinal follow-up and outcomes among a population with chronic kidney disease in a large managed care organization. Arch Intern Med 2004;164:659-663. 
-7 Haberl R, Becker A, Leber A, et al: Correlation of coronary calcification and angiographically documented stenoses in patients with suspected coronary artery disease: results of 1,764 patients. J Am Coll Cardiol 2001;37:451-457.

-8 Keelan PC, Bielak LF, Ashai K, et al: Long-term prognostic value of coronary calcification detected by electron-beam computed tomography in patients undergoing coronary angiography. Circulation 2001;104:412-417.

-9 Wexler L, Brundage B, Crouse J, et al: Coronary artery calcification: pathophysiology, epidemiology, imaging methods, and clinical implications. A statement for health professionals from the American Heart Association. Writing Group. Circulation 1996;94:1175-1192.

-10 Agatston AS, Janowitz WR, Hildner FJ, Zusmer NR, Viamonte M Jr, Detrano R: Quantification of coronary artery calcium using ultrafast computed tomography. J Am Coll Cardiol 1990;15:827-832.

-11 Kramer H, Toto R, Peshock R, Cooper R, Victor R: Association between chronic kidney disease and coronary artery calcification: the Dallas Heart Study. J Am Soc Nephrol 2005;16:507-513.

-12 Ix JH, Katz R, Kestenbaum B, et al: Association of mild to moderate kidney dysfunction and coronary calcification. J Am Soc Nephrol 2008;19:579-585.

$\checkmark 13$ Adeney KL, Siscovick DS, Ix JH, et al: Association of serum phosphate with vascular and valvular calcification in moderate CKD. J Am Soc Nephrol 2009;20:381-387.

-14 Russo D, Palmiero G, De Blasio AP, Balletta MM, Andreucci VE: Coronary artery calcification in patients with CRF not undergoing dialysis. Am J Kidney Dis 2004;44:1024-1030.

-15 Garland JS, Holden RM, Groome PA, et al: Prevalence and associations of coronary artery calcification in patients with stages 3 to 5 CKD without cardiovascular disease. Am J Kidney Dis 2008;52: 849-858.

-16 Piers LH, Touw HR, Gansevoort R, et al: Relation of aortic valve and coronary artery calcium in patients with chronic kidney disease to the stage and etiology of the renal disease. Am J Cardiol 2009; 103:1473-1477.

-17 Levey AS, Bosch JP, Lewis JB, Greene T, Rogers N, Roth D: A more accurate method to estimate glomerular filtration rate from serum creatinine: a new prediction equation. Modification of Diet in Renal Disease Study Group. Ann Intern Med 1999;130:461-470.

-18 National Kidney Foundation: K/DOQI clinical practice guidelines for chronic kidney disease: evaluation, classification, and stratification. Am J Kidney Dis 2002;39:S1-S266.

19 National Kidney Foundation: K/DOQI clinical practice guidelines for bone metabolism and disease in chronic kidney disease. Am J Kidney Dis 2003;42:S1-S201.

-20 Third Report of the National Cholesterol Education Program (NCEP) Expert Panel on Detection, Evaluation, and Treatment of High Blood Cholesterol in Adults (Adult Treatment Panel III) final report. Circulation 2002;106:3143-3421.

-21 Wilson PW, D’Agostino RB, Levy D, Belanger AM, Silbershatz H, Kannel WB: Prediction of coronary heart disease using risk factor categories. Circulation 1998;97:1837-1847.

-22 Hong C, Bae KT, Pilgram TK: Coronary artery calcium: accuracy and reproducibility of measurements with multi-detector row CT - assessment of effects of different thresholds and quantification methods. Radiology 2003;227:795-801.

-23 Goodman WG, Goldin J, Kuizon BD, et al: Coronary-artery calcification in young adults with endstage renal disease who are undergoing dialysis. N Engl J Med 2000;342:1478-1483.

-24 Stompor T, Pasowicz M, Sullowicz W, et al: An association between coronary artery calcification score, lipid profile, and selected markers of chronic inflammation in ESRD patients treated with peritoneal dialysis. Am J Kidney Dis 2003;41:203-211.

-25 Ammirati AL, Dalboni MA, Cendoroglo M, et al: The progression and impact of vascular calcification in peritoneal dialysis patients. Perit Dial Int 2007;27:340-346.

-26 Coen G, Pierantozzi A, Spizzichino D, et al: Risk factors of one year increment of coronary calcifications and survival in hemodialysis patients. BMC Nephrol 2010;11:10.

27 Tomiyama C, Higa A, Dalboni MA, et al: The impact of traditional and non-traditional risk factors on coronary calcification in pre-dialysis patients. Nephrol Dial Transplant 2006;21:2464-2471.

- 28 Seyahi N, Kahveci A, Bolayirli M, et al: Coronary artery calcification and chronically decreased GFR in living kidney donors. Am J Kidney Dis 2007;49:143-152.

-29 Fox CS, Larson MG, Keyes MJ, et al: Kidney function is inversely associated with coronary artery calcification in men and women free of cardiovascular disease: the Framingham Heart Study. Kidney Int 2004;66:2017-2021. 
-30 Qunibi WY, Abouzahr F, Mizani MR, Nolan CR, Arya R, Hunt KJ: Cardiovascular calcification in Hispanic Americans (HA) with chronic kidney disease (CKD) due to type 2 diabetes. Kidney Int 2005;68:271-277.

-31 Miller LW: Cardiovascular toxicities of immunosuppressive agents. Am J Transplant 2002;2:807-818.

-32 Weiner DE, Tighiouart H, Elsayed EF, et al: The Framingham predictive instrument in chronic kidney disease. J Am Coll Cardiol 2007;50:217-224.

- 33 Floege J, Ketteler M: Vascular calcification in patients with end-stage renal disease. Nephrol Dial Transplant 2004;19(suppl 5):V59-V66.

-34 Rashid G, Bernheim J, Green J, Benchetrit S: Parathyroid hormone stimulates endothelial expression of atherosclerotic parameters through protein kinase pathways. Am J Physiol Renal Physiol 2007; 292:F1215-F1218.

- 35 Graciolli FG, Neves KR, dos Reis LM, et al: Phosphorus overload and PTH induce aortic expression of Runx2 in experimental uraemia. Nephrol Dial Transplant 2009;24:1416-1421.

- 36 Walker MD, Silverberg SJ: Cardiovascular aspects of primary hyperparathyroidism. J Endocrinol Invest 2008;31:925-931.

-37 Hagstrom E, Hellman P, Larsson TE, et al: Plasma parathyroid hormone and the risk of cardiovascular mortality in the community. Circulation 2009;119:2765-2771.

- 38 Hagstrom E, Ingelsson E, Sundstrom J, et al: Plasma parathyroid hormone and risk of congestive heart failure in the community. Eur J Heart Fail 2010;12:1186-1192.

- 39 Tuttle KR, Short RA: Longitudinal relationships among coronary artery calcification, serum phosphorus, and kidney function. Clin J Am Soc Nephrol 2009;4:1968-1973. 